\title{
Robust RNAi-Based Resistance to Mixed Infection of Three Viruses in Soybean Plants Expressing Separate Short Hairpins from a Single Transgene
}

\author{
Xiuchun Zhang, Shirley Sato, Xiaohong Ye, Anne E. Dorrance, T. Jack Morris, Thomas E. Clemente, and Feng Qu
}

First, fourth, and seventh authors: Department of Plant Pathology, The Ohio State University, 1680 Madison Ave, Wooster 44691; first author: Key Laboratory of Tropical Crop Biotechnology, Ministry of Agriculture, Institute of Tropical Biosciences and Biotechnology, Chinese Academy of Tropical Agricultural Sciences, Haikou 571101, China; second and sixth authors: Center for Plant Science Innovation and Department of Agronomy and Horticulture, University of Nebraska, Lincoln 68588; and third and fifth authors: Nebraska Center for Virology and School of Biological Sciences, University of Nebraska, Lincoln 68583.

Accepted for publication 3 May 2011.

\begin{abstract}
Zhang, X., Sato, S., Ye, X., Dorrance, A. E., Morris, T. J., Clemente, T. E., and Qu, F. 2011. Robust RNAi-based resistance to mixed infection of three viruses in soybean plants expressing separate short hairpins from a single transgene. Phytopathology 101:1264-1269.

Transgenic plants expressing double-stranded RNA (dsRNA) of virus origin have been previously shown to confer resistance to virus infections through the highly conserved RNA-targeting process termed RNA silencing or RNA interference (RNAi). In this study we applied this strategy to soybean plants and achieved robust resistance to multiple viruses with a single dsRNA-expressing transgene. Unlike previous reports that relied on the expression of one long inverted repeat (IR) combining sequences of several viruses, our improved strategy utilized a transgene designed to express several shorter IRs. Each of these short IRs contains highly con-

short dsRNA stems were interspersed with single-stranded sequences to prevent homologous recombination during the transgene assembly process. Three such short IRs with sequences of unrelated soybean-infecting viruses (Alfalfa mosaic virus, Bean pod mottle virus, and Soybean mosaic virus) were assembled into a single transgene under control of the $35 \mathrm{~S}$ promoter and terminator of Cauliflower mosaic virus. Three independent transgenic lines were obtained and all of them exhibited strong systemic resistance to the simultaneous infection of the three viruses. These results demonstrate the effectiveness of this very straight forward strategy for engineering RNAi-based virus resistance in a major crop plant. More importantly, our strategy of construct assembly makes it easy to incorporate additional short IRs in the transgene, thus expanding the spectrum of virus resistance. Finally, this strategy could be easily adapted to control virus problems of other crop plants.
\end{abstract} served sequences of one virus, forming dsRNA of less than $150 \mathrm{bp}$. These
Virus diseases cause significant losses in the yield and quality of soybean seeds on an annual basis. Serious, early season virus infections frequently result in stunted plants and deformed seed pods which result in yield loss. Milder, later season infections often cause discoloration of soybean seeds (seed coat mottling) that affects the commercial value of the seeds (7). The three most important viral pathogens of soybean in United States are Alfalfa mosaic virus (AMV), a member of the family Bromoviridae, Bean pod mottle virus (BPMV), a member of the family Secoviridae, and Soybean mosaic virus (SMV), a member of the family Potyviridae. These viruses belong to different virus families, have distinctive modes of transmission, and use different strategies to achieve successful reproduction in plants. These differences have complicated efforts to breed for multiple virus resistance. Indeed, only SMV has been effectively controlled using commercially available resistant soybean varieties (16).

Research during the last decade has firmly established that land plants use an RNA-targeting defense mechanism termed RNA silencing (or RNA interference, RNAi) to combat virus infections $(5,13)$. RNAi uses double-stranded RNA (dsRNA) produced dur-

Corresponding authors: F. Qu; E-mail address: qu.28@osu.edu

T. E. Clemente; E-mail address: tclemente1@unl.edu

* The $\boldsymbol{e}$-Xtra logo stands for "electronic extra" and indicates that Figure 2 appears in color online.

doi:10.1094/PHYTO-02-11-0056

(C) 2011 The American Phytopathological Society ing the intracellular replication process of viruses as template to produce small RNAs (vsRNAs) of 21 to 24 nucleotides (nt) long. vsRNAs then target homologous viral RNAs for degradation or translational repression in a sequence-specific manner. In most virus infections RNA silencing is at least partially suppressed by silencing suppressors encoded by viruses. Nevertheless, previous studies have demonstrated that plants can gain a competitive advantage against invading viruses if they are programmed to produce vsRNAs ahead of virus invasion $(2,10,19)$. This is most frequently achieved by transgenically expressing portions of a virus genome, which are converted into dsRNAs and subsequently vsRNAs by the plant RNAi machinery.

Two previous studies have successfully engineered resistance in soybean to two different viruses-BPMV and Soybean dwarf virus (SbDV) - by incorporating the coding sequences of viral capsid proteins (CPs) into the transgene $(15,17)$. In the first study, Reddy and colleagues (15) expressed the coding sequence for the two CP subunits of BPMV, which remain as one protein in the absence of virus-encoded protease. Consequently, a protein of expected size was detected in the transgenic soybean plants. However, it is unclear whether RNAi played a major role in this case as the authors did not determine whether resistance correlated with vsRNA-mediated degradation of viral or transgene RNA (14). On the other hand, the SbDV resistance observed by Tougou and colleagues (17) was due primarily to RNAi activity as the SbDV-specific transgene contained an inverted repeat (IR) of SbDV CP coding sequence, which is known to be a strong inducer of RNAi. Indeed, vsRNAs were readily detectable in the trans- 
genic plants (17). These studies proved that transgenic expression of viral genome segments can be used to achieve effective control of virus diseases in soybean. However, the usefulness of the above mentioned examples is limited by a number of drawbacks. First, the viral $\mathrm{CP}$ sequences included in the transgenes are considered to be more prone to genetic variations (9). Thus, resistance conferred by CP-targeting transgenes may be effective to a limited range of virus strains. In addition, both of the above-mentioned transgenic plants were designed to target one virus $(15,17)$. This transgene design is less desirable since it is unable to counter mixed infections of several viruses often associated with fieldgrown soybean.

In this report, we describe a relatively simple transgenic approach that allowed us to effectively control AMV, BPMV, and SMV simultaneously, even when they were introduced into the transgenic plants at the same time. Unlike previous studies, we created a transgene construct that is composed of three short IRs, each containing the specific, highly conserved sequences derived from one virus. These short IR modules were separated by various lengths of nonrepeating sequences, alleviating the potential of insert instability associated with assembling longer IRs. Furthermore, this modular design allows additional modules to be stacked onto the same construct with relative ease, thus expanding the resistance spectrum of the construct. Our results indicate that dsRNA regions as short as $100 \mathrm{bp}$ can serve as efficient templates for vsRNA biogenesis, leading to robust systemic virus resistance.

\section{MATERIALS AND METHODS}

Assembly of the transgene. The transgene construct, dsABS, was designed to express three short RNA hairpins in transgenic plants, each with a double-stranded region of 100 to $150 \mathrm{bp}$ and an end loop of about $100 \mathrm{nt}$. The sequences of these hairpins were derived from the highly conserved regions of the replicase genes of AMV, BPMV, and SMV, respectively (Fig. 1). Specifically, the AMV-based hairpin (dsA) is composed of nucleotides 1809 to 2063 [in viral (+) sense orientation] and 1950 to 1842 of AMV
RNA2 [in complementary (-) sense orientation], resulting in a 109-bp dsRNA (1842 to 1950) region, and a $113 \mathrm{nt}$ (1951 to 2063) end loop. Similarly, the BPMV-based hairpin (dsB) is made of nucleotides 4507 to 4770 [(+) orientation] and 4677 to 4531 [(-) orientation] of BPMV RNA1, resulting in a 147-bp dsRNA (4531 to 4677) region and a 93 nt (4678 to 4770) end loop. Finally, the SMV-based hairpin (dsS) contains nucleotides 7408 to $7661[(+)$ orientation] and 7587 to $7465[(-)$ orientation] of SMV RNA, folding into a dsRNA region of $123 \mathrm{bp}$ (7465 to 7587) and an end loop of $74 \mathrm{nt}$ (7588 to 7661). The sequences of DNA oligos used to produce the above fragments are available upon request.

The cDNAs of the three hairpins were linked together in the order of dsABS, and cloned between the Cauliflower mosaic virus (CaMV) 35S promoter (P35S) and terminator (T35S) in the plasmid pRTL2 (3; with modified multicloning sites). The entire P35S-dsABS-T35S cassette was then mobilized into the binary vector pPTN200. The resulting construct, pPTN-dsABS, was transformed into Agrobacterium tumefaciens (strain EHA101).

Soybean transformation. The soybean cultivar Throne was transformed as previously described (4). The transgenic plants were first screened by rubbing a $100 \mu \mathrm{g} / \mathrm{ml}$ solution of glufosinate ammonium on the surface of one of mono-foliate leaves of T0 seedlings. Progenies of herbicide-resistant transformants were further confirmed using several different procedures. First, they were inoculated with AMV, BPMV, and SMV, individually and in combination, to evaluate their resistance to the viruses. Second, they were subject to polymerase chain reaction (PCR)-based genotyping to verify the presence of the herbicide resistance (BAR) gene. Third, the plants were examined for the presence of small interfering RNAs (siRNAs) of AMV, BPMV, and SMV origins, which are the expected products of the short hairpin transgenes.

Enzyme-linked immunosorbent assay. Eenzyme-linked immunosorbent assay (ELISA) kits complete with anti-AMV, antiBPMV, or anti-SMV antibodies were purchased from Agdia Inc. (Elkhart, IN) and the detections were carried out according to manufacturer's instructions. To ensure reproducibility of ELISA

AMV RNA2:

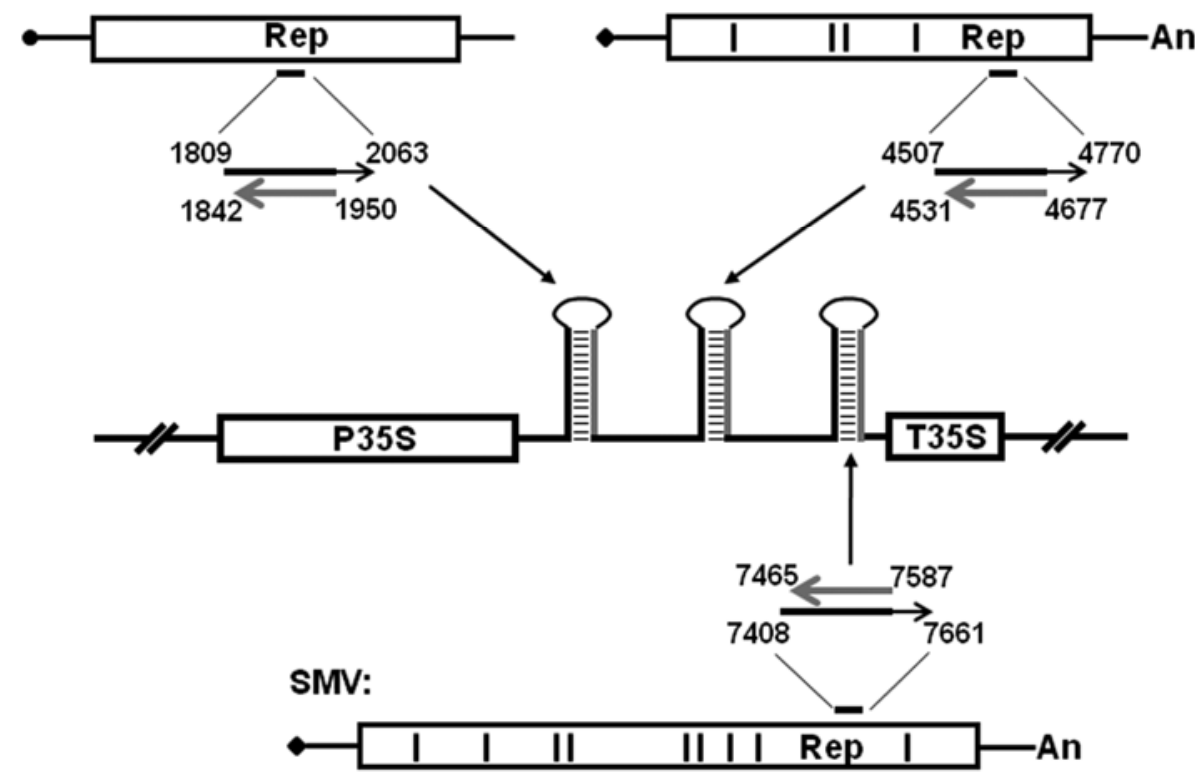

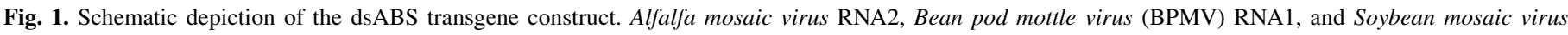

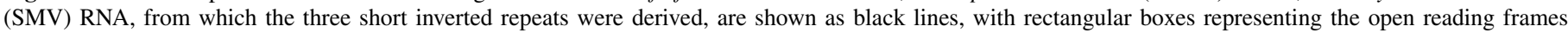

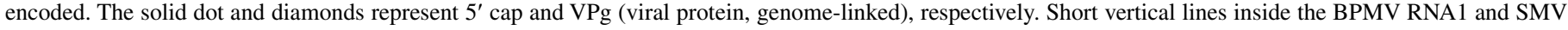

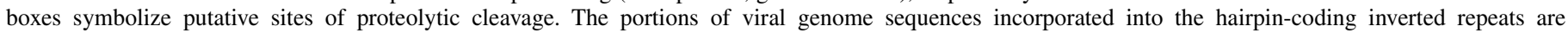

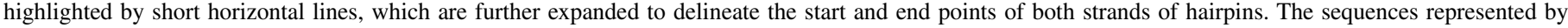

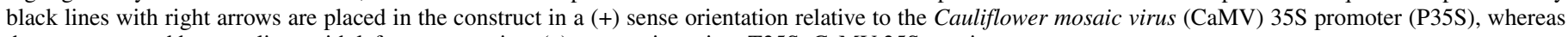
those represented by gray lines with left arrows are in a (-) sense orientation. T35S: CaMV 35S terminator. 
results, we collected samples from five or more plants in every treatment group for testing. The raw ELISA readings were then averaged and converted to multiples of negative controls (inoculated with buffer only) using the following formula: (sample blank)/(negative control - blank). Any samples with an adjusted value of 2.0 or above were considered positive for the corresponding virus.

PCR-based genotyping. The REDExtract-N-Amp Plant PCR kit (Sigma, Saint Louis, MO) and the Terra PCR Direct Polymerase mix (Clontech, Palo Alto, CA) were used alternately to genotype soybean plants with a pair of primers specific for the BAR reporter gene (sequences of primers available upon request).

Reverse transcription-PCR. Total RNAs were extracted from both mock-inoculated and virus (AMV, BPMV, and SMV)-infected plants at 5 weeks postinoculation (wpi) using the TRIsure reagent (Bioline USA, Tauton, MA). The total RNA samples were then treated with RNase-free DNase, reverse-transcribed with virus-specific primers. Virus-specific cDNA were then PCR-amplified using the following primers: AMVR2-1139F (CTG AAG CCC TAT TAG CGA TAA AGA AAC) and AMVR2-1757R (CTA TGC GCA TTA AAC CAT AAG GTT AGA) for AMV; BPMVR2-669F (GCA ACA TTC ATA TTT CTG GCT TGT TCT) and BPMVR2-1425R (GCA GCA CAT GCT ACA TTA ACC TTG A) for BPMV; and SMV-7311F (GTG TAT GTG ACT GAT CCT GAT GA) and SMV-7828R (AGC ATT TCT CTT CCA ACC CAC CA) for SMV. Primers specific for the soybean actin gene were used to amplify actin-specific cDNA fragments, serving as positive controls.

Detection of vsRNAs using RNA blot hybridization. Total RNA were extracted as described above. Twenty-five micrograms of each total RNA sample was separated on a $16 \%$ polyacrylamide, $0.1 \times$ TBE, $8 \mathrm{M}$ urea gel, transferred to nylon membranes, and subjected to hybridizations with a ${ }^{32} \mathrm{P}$-labeled probe consisting of three PCR fragments homologous to the three inverted repeats in the dsABS transgene (nucleotides 1842 to 1950 of AMV RNA2, nucleotides 4531 to 4677 of BPMV RNA1, and nucleotides 7465 to 7587 of SMV). The PerfectHyb Plus hybridization buffer was purchased from Sigma. The detailed protocol has been described elsewhere (14).

\section{RESULTS}

Three independent lines of transgenic soybean containing the dsABS transgene are resistant to simultaneous challenges of BPMV and SMV. In order to achieve the goal of controlling the three major soybean-infecting viruses with one transgene

TABLE 1. Presence of viruses in T1 generation dsABS plants infected with Bean pod mottle virus (BPMV) + Soybean mosaic virus (SMV) at 2 and 5 weeks postinoculation (wpi) as measured using enzyme-linked immunosorbent assay

\begin{tabular}{lccccc}
\hline Line & Inoculum & $\begin{array}{c}\text { BPMV, } \\
2 \text { wpi }\end{array}$ & $\begin{array}{c}\text { BPMV, } \\
5 \text { wpi }\end{array}$ & $\begin{array}{c}\text { SMV, } \\
2 \text { wpi }\end{array}$ & $\begin{array}{c}\text { SMV, } \\
5 \text { wpi }\end{array}$ \\
\hline NT & BPMV+SMV & $>10(+)$ & $>10(+)$ & $>10(+)$ & $>10(+)$ \\
$729-3$, T1 & BPMV+SMV & $9.77(+)$ & $1.9(-)$ & $1.1(-)$ & $0.9(-)$ \\
NT & BPMV+SMV & $>10(+)$ & $>10(+)$ & $>10(+)$ & $>10(+)$ \\
$729-4$, T1 & BPMV+SMV & $6.6(+)$ & $1.5(-)$ & $0.87(-)$ & $0.3(-)$ \\
NT & BPMV+SMV & $>10(+)$ & N/A & $>10(+)$ & N/A \\
$729-5$, T1 & BPMV+SMV & $2( \pm)$ & N/A & $0.4(-)$ & N/A \\
\hline
\end{tabular}

a The numbers in the last four columns are relative values calculated as follows. First, the average reading of five blank wells of every plate was deducted from the average readings of both negative controls (samples collected from five mock-inoculated plants) and actual samples (at least five for every treatment) to obtain the adjusted readings of negative controls and actual samples. Then, the adjusted readings of actual samples were divided by that of negative controls. A relative value of 2.0 or above indicates the presence of the respective virus in the samples examined [denoted by $(+)$ ]. NT: nontransgenic plants. N/A: not assessed. construct, we decided to assemble an RNAi construct composed of three separate IRs, each designed to express a short hairpin targeting one virus. To ensure this construct confers resistance to a maximum number of strains of the target viruses, we first identified the highly conserved regions from the replicase genes of AMV, BPMV, and SMV. This is accomplished by comparing all strains of each virus whose sequences are available through GenBank. Consequently, the 109 nt AMV sequence that forms the first dsRNA stem in the dsABS construct (Fig. 1) is at least $93 \%$ identical to the four AMV RNA2 sequences deposited in GenBank. Similarly, the $147 \mathrm{nt}$ sequence of the BPMV hairpin stem is at least $97 \%$ identical to the six BPMV RNA1 sequences found in the database. Remarkably, the $123 \mathrm{nt}$ long SMV hairpin stem is at least $94 \%$ identical with 53 available SMV sequences.

The identified sequences were assembled into the dsABS transgene as depicted in Figure 1 and detailed in the Materials and Methods section. Transgenic plants were generated from cotyledons of Thorne variety of soybean using a previously described procedure (4). Initial screening by rubbing a diluted glufosinate solution $(100 \mu \mathrm{g} / \mathrm{ml})$ on leaves identified three lines, 729-3, 729-4, and $729-5$, as containing the transgene of interest. Seeds harvested from the three primary transformants were planted, and the T1 plants were rescreened. For the purpose of determining the number of transgene loci in each line, descendants of all T1 lines were genotyped again with a PCR procedure (see Materials and Methods for details). A total of over 100 individual plants were genotyped for each line. The number of plants that inherited the transgene is 81 out of a total of 126 for line 729-3, 93 out of 122 for line 729-4, and 78 out of 108 for line 729-5. We conclude from these data that the dsABS transgene segregated at a ratio of roughly $3: 1$ for all three lines, suggesting that they each harbor a single transgene copy.

The transgenic T1 plants were divided into two groups with roughly equal number of plants (usually five to nine plants in each group). Plants in the first group were reserved for seed-setting as well as serving as mock-inoculated controls during the first round of screening for virus resistance. The second group of T1 plants were inoculated with a mixed inoculum containing BPMV and SMV and assessed for resistance to systemic spread of both viruses. Most inoculated plants showed viral symptoms at about 2 wpi, although the symptoms in the transgenic plants were milder than in their nontransgenic (NT) counterparts. By 5 wpi, the transgenic plants became largely symptomless, whereas their NT counterparts remained symptomatic (data not shown). Accordingly, the height and canopy circumference of the virusinoculated transgenic plants were about the same as mock-inoculated controls. In contrast, all of the virus-inoculated NT plants were severely stunted (data not shown).

We further evaluated the titers of BPMV and SMV in the inoculated plants using ELISA, with virus-specific antibodies (Agdia, Elkhart, IN). Plants were identified as susceptible to the assayed virus when the ELISA readings of their tissues were at least twofold higher than those of mock-inoculated controls (Table 1). At 2 wpi, BPMV, but not SMV, was detected at high levels in lines 729-3 and 729-4, but at very low levels in line 729-5 (Table 1). By 5 wpi, the virus titers were below the detection limit for both viruses in all three dsABS lines (Table 1; and data not shown). We conclude from these results that immediately after inoculations, both viruses were able to replicate in the transgenic plants. However, the initial replication was countered by a defense mechanism that leads to the clearance of viruses within a relative short period. Hence, the expression of the short hairpin transgene in the transgenic dsABS plants conferred a strong RNAi-based resistance response to both BPMV and SMV. These encouraging results prompted us to further evaluate the T2 seeds for resistance to all three viruses.

All dsABS transgenic plants display robust resistance to simultaneous inoculations of AMV, BPMV, and SMV. To 
further evaluate if the 729-3, 729-4, and 729-5 lines were resistant to all three viruses targeted by the dsABS transgene, we selected the T2 progenies of four T1 lines (729-3, T1-10; 729-4, T1-4; 729-5, T1-4 and T1-8) for additional detailed analyses. Seeds from these four $\mathrm{T} 1$ lines were germinated, and transgene-containing individuals were identified using a PCR-based genotyping procedure. They were then inoculated with a mixed inoculum containing AMV, BPMV, as well as SMV. NT soybean plants (Thorne variety) were used as controls.

The four transgenic lines displayed consistent results. They all proved to be highly resistant to mixed infection of three viruses. Figure 2A shows a typical set of plants illustrating the contrasting symptoms on NT and dsABS-transgenic plants (line 729-3, T1-10; photographed at $6 \mathrm{wpi}$ ). The NT control plants were visibly stunted with severe symptoms of systemic virus infection. By contrast, virus-inoculated dsABS plants were indistinguishable from the mock-inoculated NT or dsABS plants. The symptoms are shown for only one of the lines tested but similar results were obtained for all lines tested.

AMV, BPMV, and SMV genomic RNAs are cleared from the dsABS plants. To confirm that the recovery from initial disease symptoms in dsABS plants was due to the clearance of viral genomic RNAs, we extracted total RNAs from young leaves of the inoculated plants at 5 wpi. The samples were then subjected to reverse transcription-PCR analyses using primers specific for AMV, BPMV, and SMV, respectively. As shown in Figure 2B,
AMV-, BPMV-, and SMV-specific PCR products were easily detectable in virus-infected NT plants (lanes 5 and 6 of all three panels), but not in dsABS-transgenic plants. These results provide convincing evidence that viral RNAs of all three viruses were cleared from the systemic leaves of the transgenic plants.

The presence of dsABS transgene prevents virus-induced seed mottling. Later season virus infections in soybeans often results in milder symptoms that may not cause a significant yield loss. However, these plants can often produce discolored seeds which have a reduced commercial value. As shown above, our dsABS transgenic lines initially showed moderate symptoms that soon dissipated, raising the possibility that these plants might not escape the seed mottling damage. In view of this, we harvested seed from all of the infected plants to inspect for seed mottling. As shown in Figure 2C, while a high proportion of seeds from virus-infected NT plants were small and discolored (50 to 60\%), none of the seeds from the dsABS counterparts could be distinguished from the healthy seeds of the mock-inoculated control plants. These results clearly demonstrate that the dsABS transgene not only prevented the disease symptoms on the developing plants, but also eliminated seed mottling associated with virus infections.

Accumulation of vsRNAs in dsABS plants is enhanced by virus infection. The short IRs of virus origins in the dsABS transgene would be expected to be transcribed into RNAs capable of folding into three short hairpin RNAs, which in turn are

A

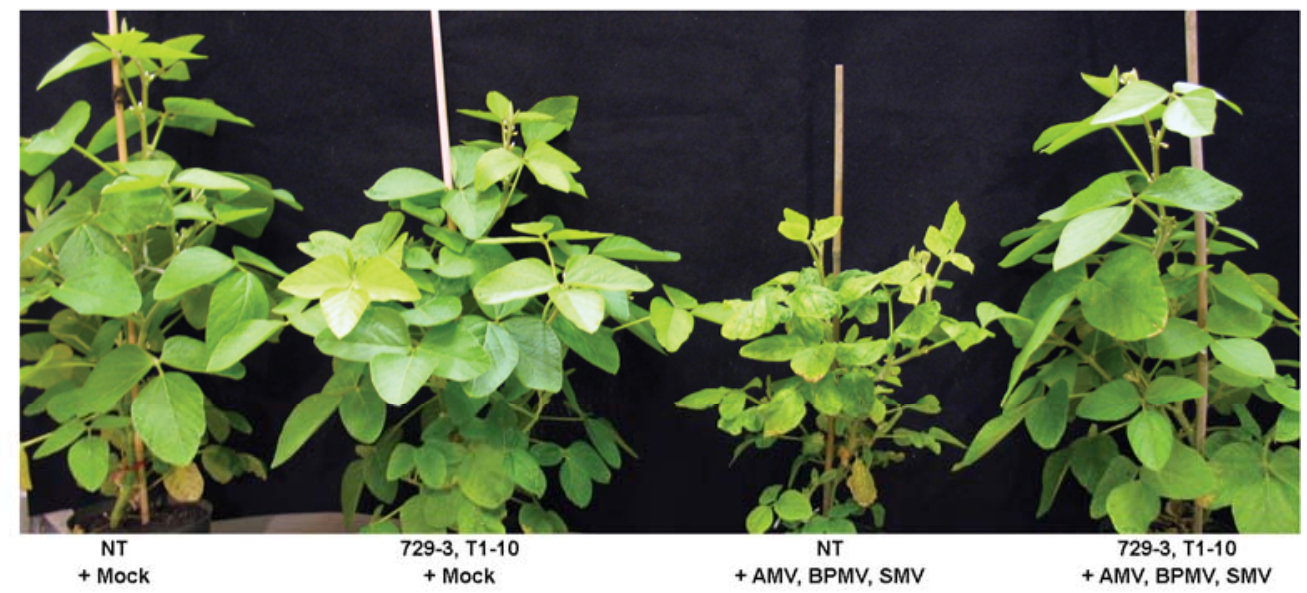

B

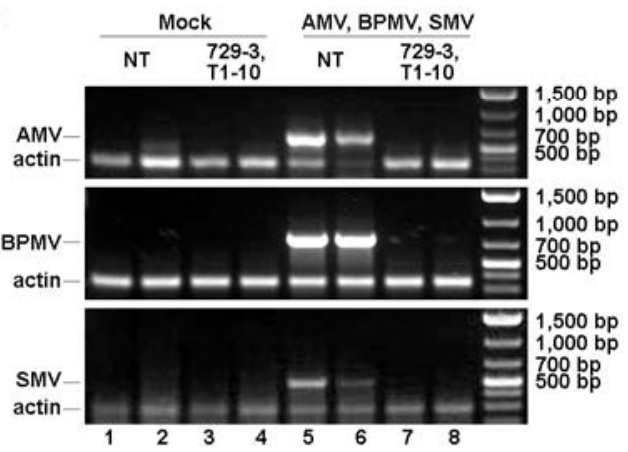

C

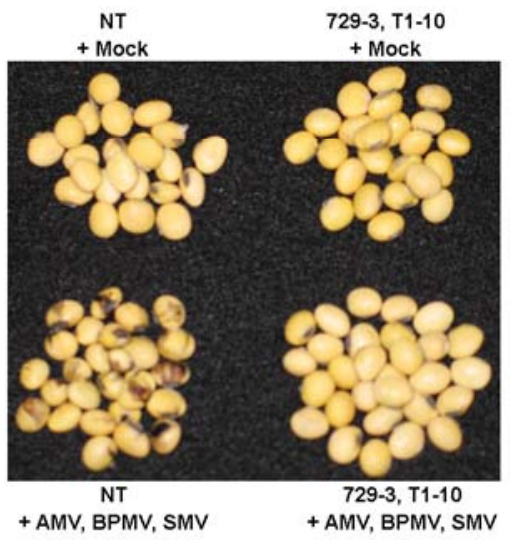

Fig. 2. The dsABS transgenic plants are highly resistant to systemic invasion after mechanical inoculation with Alfalfa mosaic virus (AMV), Bean pod mottle virus (BPMV), and Soybean mosaic virus (SMV). The progenies of line 729-3, T1-10 are illustrated and representative of all three transgenic soybean lines which displayed similar levels of resistance. A, Symptoms on AMV-, BPMV-, and SMV-infected nontransgenic (NT) and dsABS-transgenic (729-3, T1-10) plants 6 weeks after mechanical inoculation with all three viruses (6 weeks postinoculation). Mock-inoculated nontransgenic and transgenic plants were used as controls. B, Reverse transcription-polymerase chain reaction (PCR) results confirming the absence of AMV, BPMV, and SMV RNAs in inoculated dsABS plants. The expected sizes of PCR fragments are 618 bp for AMV (nucleotides 1139 to 1757 of AMV RNA2), 756 bp for BPMV (nucleotides 669 to 1425 of BPMV RNA2), and 517 bp for SMV (nucleotides 7311 to 7828 of SMV RNA). The soybean actin mRNA was used as a positive control. C, Sample of seeds harvested from mature plants grown in the greenhouse. Note the presence of extensive seed mottling on the virus-inoculated control seed compared with the resistant line 729-3. 
processed into virus-targeting vsRNAs. To confirm that vsRNAs are indeed present in the transgenic plants, we sought to detect vsRNAs in the transgenic plants using RNA blot hybridizations. Since all four selected lines gave rise to similar results, we again chose to present the blot of line 729-3, T1-10. As shown in Figure 3 , vsRNAs of 21,22 , and $24 \mathrm{nt}$ in size were detected in mockinoculated dsABS-transgenic plants but not in NT controls (compare lanes 3 and 4 with 1 and 2), indicating that the dsRNA expressed from the transgene were processed into vsRNAs. As expected, virus-specific vsRNAs were also produced in virusinfected NT plants at low levels (lanes 5 and 6), indicating that RNA genomes of invading viruses also served as substrates for vsRNA biogenesis. Interestingly, virus infection drastically increased the accumulation levels of vsRNAs in the dsABS transgenic plants. This result suggests that the transgene-derived vsRNAs likely served as the primary vsRNAs that primed the amplification of vsRNAs using input viral RNAs as templates. Together, our results convincingly demonstrate that engineering soybean plants to express virus-targeting short hairpin RNAs is an effective virus control strategy that could have broader application potentials for managing virus problems in other economically important crop plants.

\section{DISCUSSION}

Soybean production is frequently hampered by virus infections, a problem often exacerbated by relatively warm spring weather that promotes virus-vectoring insects to populate and feed on young seedlings. Conventional control measures aimed at managing virus vectors have had limited success (1). Although virusresistant soybean varieties are available to protect against a limited number of viruses, their production is expensive and it often takes years to breed resistance into desirable soybean varieties (16). Here, we report a relatively simple genetic engineering approach that offers a promising alternative for controlling most major virus disease problems in soybeans. To summarize, we have assembled into a single transgene three short IRs that target conserved genome sections of three different viruses (AMV, BPMV, and SMV). Transgenic plants expressing the transgene produce vsRNAs specific for the three viruses, and are highly resistant to these viruses, blocking the spread of systemic symptoms and eliminating seed mottling. This report represents the first demonstration of the applicability of a facile RNAi strategy

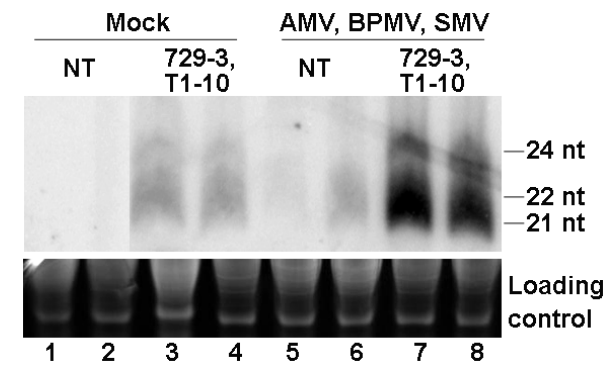

Fig. 3. Detection of vsRNAs in dsABS transgenic plants. Plants of transgenic line 729-3 (descendants of T1-10), together with nontransgenic (NT) control plants, were mechanically inoculated with buffer only (mock), or with a mixed inoculum containing Alfalfa mosaic virus (AMV), Bean pod mottle virus (BPMV), and Soybean mosaic virus (SMV). Total RNAs isolated from young uninoculated leaves at 5 weeks postinoculation were separated on a $16 \%$ polyacrylamide gel electrophoresis, $8 \mathrm{M}$ urea, and $0.1 \times \mathrm{TBE}$, and transferred to nylon membranes. The hybridization was carried out at $42^{\circ} \mathrm{C}$ in the PerfectHyb Plus buffer (Sigma-Aldrich, St. Louis, MO). The probe used was a mixture of three polymerase chain reaction (PCR) fragments that correspond to the double-stranded RNA regions of the three short hairpins in the dsABS construct (nucleotides 1842 to 1950 of AMV RNA2, nucleotides 4531 to 4677 of BPMV RNA1, and nucleotides 7465 to 7587 of SMV), labeled with ${ }^{32} \mathrm{P}$ through random priming. Using the three PCR fragments separately yielded weaker signals but of essentially the same pattern (data not shown). to engineer antiviral defense to multiple viruses of choice in commercial soybean.

Several previous studies reported the use of RNAi-based strategies to engineer multivirus resistance in transgenic plants $(2,10,19)$. However, our approach differs significantly from these previous studies. In studies cited above, a long virus-derived fragment (600 to 1,000 bp) was first assembled from segments of different viruses. This long fragment was then placed on both sides of an intron in opposite directions such that the RNA transcribed from the transgene would form a single, long dsRNA after intron removal. The presence of the long IRs could pose potential problems in the process of construct assembly as they are prone to homologous recombination, leading to truncation or complete loss of the insert. Indeed, we have noticed in our unpublished experiments that the number of bacterial colonies containing correct recombinant plasmids drops precipitously as the size of IRs increased. In addition, the yield of recombinant plasmids also dropped to very low levels, making the construction of such recombinant plasmids a genuine challenge.

By contrast, our transgene construct is composed of three independent short IRs that are separated by nonrepeat regions of similar lengths. Furthermore, most of the nonrepeated regions, including those in the loop regions of the expected hairpins, as well as those separating the three independent IRs, were sequences of virus origin. Therefore, even though the actual repeats destined for dsRNA formation are relatively short in our construct (less than $150 \mathrm{bp}$ ), much longer regions of viral RNA could be potentially targeted by the secondary vsRNAs produced from the single-stranded portions of the transgene. The results reported here, together with our unpublished data using numerous independent transformants consisting of two short hairpins (targeting BPMV and SMV), illustrate that close to $100 \%$ of the transgenic lines generated using our strategy were effective at conferring virus resistance. Our next step will be to assess the effectiveness of dsABS-transgenic plants in field conditions, where the capability of the transgene to counter insect-transmitted virus invasions will be evaluated.

Engineering virus resistance in soybean using virus-derived sequences has been reported before $(15,17,18)$. Compared with these earlier studies, our transgene design has at least two advantages. First, instead of using viral $\mathrm{CP}$ coding sequence to construct the transgene, we chose to use highly conserved regions of viral replicase genes. Viral CPs are considered to be more variable than viral replicases (9). As a result, RNAi triggered by $\mathrm{CP}$ coding sequence likely will target a limited number of closely related strains of a given virus. By contrast, RNAi triggered by highly conserved replicase sequences should be effective against most strains of a virus. The dsABS transgene used in the current study combined carefully selected regions of AMV, BPMV, and SMV replicase genes that are at least $93 \%$ identical to all known strains of respective viruses at the nucleotide level. Based on a recent report showing that $88 \%$ of sequence identity was sufficient to confer highly effective RNAi-triggered virus resistance, we predict that the dsABS transgenic plants can be used to combat a wide range of virus strains (6).

Notably, the antiviral resistance conferred by the dsABS transgene was reinforced by infections with target viruses, leading to a complete recovery of the infected plants after a brief period of symptomatic infections. Considering the relatively short lengths of IR regions in the dsABS transgene, it is of interest to evaluate whether longer IRs would further enhance the resistance level to full immunity.

Another advantage of our transgene design is that it confers resistance to three different viruses with one single transgene. More importantly, thanks to the modular nature of the short hairpins, additional modules can be easily stacked to the existing construct to expand its resistance spectrum. Clearly, this approach would be easily transferable to other crop plants such as tomato, 
in which multiple viruses frequently coexist (8). It is also expected to have applications in combating other plant pathogens given several recent reports showing the effectiveness of RNAibased resistance against fungi, nematodes, and insects $(11,12)$.

\section{ACKNOWLEDGMENTS}

We thank members of our labs, P. Redinbaugh, L. Stewart, and other members of the USDA corn and soybean research group for stimulating discussions and generous sharing of equipment. This study is supported in part by funds from North Central Soybean Research Program and Ohio Soybean Council. X. Ye and T. J. Morris received funding from NIH grant P20 RR016469.

\section{LITERATURE CITED}

1. Bradshaw, J. D., Rice, M. E., and Hill, J. H. 2008. Evaluation of management strategies for bean leaf beetles (Coleoptere: Chrysomelidae) and Bean pod mottle virus (Comoviridae) in soybean. J. Econ. Entomol. 101:1211-1227.

2. Bucher, E., Lohuis, D., van Poppel, P. M. J. A., Geerts-Dimitriadou, C., Goldbach, R., and Prins, M. 2006. Multiple virus resistance at a high frequency using a single transgene construct. J. Gen. Virol. 87:3697-3701.

3. Carrington, J. C., and Freed, D. D. 1990. Cap-independent enhancement of translation by a plant potyvirus $5^{\prime}$ nontranslational region. J. Virol. 64:1590-1597.

4. Clemente, T. E., LaValle, B. J., Howe, A. R., Ward, D. C., Rozman, R. J., Hunter, P. E., Broyles, D. L., Kasten, D. S., and Hinchee, M. A. 2000. Progeny analysis of glyphosate selected transgenic soybeans derived from Agrobacterium-mediated transformation. Crop Sci. 40:797-803.

5. Ding, S.-W. 2010. RNA-based antiviral immunity. Nat. Rev. Immunol. 10:632-641.

6. Gaba, V., Rosner, A., Maslenin, L., Leibman, D., Singer, S., Kukurt, E., Shiboleth, Y. M., and Gal-On, A. 2010. Hairpin-based virus resistance depends on the sequence similarity between challenge virus and discrete, highly accumulating siRNA species. Eur. J. Plant Pathol. 128:153-164.

7. Giesler, L. J., and Ziems, A. D. 2006. Incidence of Alfalfa mosaic virus, Bean pod mottle virus and Soybean mosaic virus in Nebraska soybean fields. Plant Health Progress doi:10.1094/PHP-2006-0424-01-HM.
8. Hanssen, I. M., Lapidot, M., and Thomma, B. P. H. J. 2010. Emerging viral diseases of tomato crops. Mol. Plant-Microbe Interact. 23:539-548.

9. Hull, R. 2002. Matthew's Plant Virology, Academic Press, San Diego, CA.

10. Kreuze, J. F., Klein, I. S., Lazaro, M. U., Chuquiyuri, W. J. C., Morgan, G. L., Mejía, P. G. C., Ghislain, M., and Valkonen, J. P. T. 2008. RNA silencing-mediated resistance to a crinivirus (Closteroviridae) in cultivated sweetpotato (Ipomoea batatas L.) and development of sweetpotato virus disease following co-infection with a potyvirus. Mol. Plant Pathol. 9:589-598

11. Nowara, D., Gay, A., Lacomme, C., Shaw, J., Ridout, C., Douchkov, D., Hensel, G., Kumlehn, J., and Schweizera, P. 2010. HIGS: Host-induced gene silencing in the obligate biotrophic fungal pathogen Blumeria graminis. Plant Cell 22:3130-3141.

12. Paldi, N., Glick, E., Oliva, M., Zilberberg, Y., Aubin, L., Pettis, J., Chen, Y., and Evans, J. D. 2010. Effective gene silencing in a Microsporidian parasite associated with honeybee (Apis mellifera) colony declines. Appl. Environ. Microbiol. 76:5960-5964.

13. Qu, F. 2010. Plant viruses versus RNAi: Simple pathogens reveal complex insights on plant anti-microbial defense. Wiley Interdisciplinary Reviews: RNA (WIREs RNA). 1:22-33.

14. Qu, F., Ye, X., and Morris, T. J. 2008. Arabidopsis DRB4, AGO1, and AGO7 participate in a DCL4-initiated antiviral RNA silencing pathway that is negatively regulated by DCL1. Proc. Natl. Acad. Sci. USA 105:14732-14737.

15. Reddy, M. S. S., Ghabrial, S. A., Redmond, C. T., Dinkins, R. D., and Collins, G. B. 2001. Resistance to Bean pod mottle virus in transgenic soybean lines expressing the capsid polyprotein. Phytopathology 91:831-838.

16. Shi, A., Chen, P., Li, D., Zheng, C., Zhang, B., and Hou, A. 2009. Pyramiding multiple genes for resistance to soybean mosaic virus in soybean using molecular markers. Mol. Breed. 23:113-124.

17. Tougou, M., Furutani, N., Yamagishi, N., Shizukawa, Y., Takahata, Y., and Hidaka, S. 2006. Development of resistant transgenic soybeans with inverted repeat-coat protein genes of soybean dwarf virus. Plant Cell Rep. 25:1213-1218

18. Tougou, M., Yamagishi, N., Furutani, N., Shizukawa, Y., Takahata, Y., and Hidaka, S. 2007. Soybean dwarf virus-resistant transgenic soybeans with the sense coat protein gene. Plant Cell Rep. 26:1967-1975.

19. Zhu, C. X., Song, Y.-Z., Yin, G.-H., and Wen, F.-J. 2009. Induction of RNA-mediated multiple virus resistance to Potato virus Y, Tobacco mosaic virus, and Cucumber mosaic virus. J. Phytopathol. 157:101-107. 